\title{
Original
}

\section{Consecuencias del estrés de rol}

\section{Consequences of role stress}

\author{
Fernando Mansilla Izquierdo \\ Madrid-Salud. Madrid. España \\ Recibido: 05-10-11 \\ Aceptado: 11-10-11

\section{Correspondencia} \\ Fernando Mansilla Izquierdo \\ C/ Emilio Coll, 22, 1 \\ 28224- Pozuelo de Alarcón (Madrid). España. \\ E-mail: mansillaif@munimadrid.es
}

Resumen

El estrés de rol es específicamente el que se origina por el desempeño de roles en la organización, y comprende tanto la ambigüedad de rol como el conflicto de rol.

Objetivo: Evaluar la ambigüedad y el conflicto de rol de un grupo de profesionales que realizan diferentes puestos de trabajo, y su influencia sobre sus niveles de bienestar físico y psicológico.

Material y método: Para evaluar los estresores de rol (Ambigüedad de Rol y Conflicto de Rol) se ha utilizado el Cuestionario de Rizzo, House y Lirtzman. Y para establecer las consecuencias el Cuestionario de Síntomas SLC-90-R.

Resultados: El 20\% de la muestra percibía ambigüedad y conflicto de rol en su trabajo. Y formaron el grupo que puntuó alto en ambigüedad y conflicto de rol puntuó asimismo alto en todas las subescalas: Somatización, de Obsesión-Compulsión, de Sensibilidad Interpersonal, Depresión, Ansiedad, Hostilidad, Ansiedad Fóbica, Ideación Paranoide y de Psicoticismo. Además el Índice Global de Gravedad y el Total de Síntomas Positivos fueron significativamente superiores en el grupo que puntuó alto en ambigüedad de rol y conflicto de rol.

Por otro lado, no se encuentran diferencias significativas entre los niveles de estudios ni en las subescalas ni en Índice General de Gravedad ni en el Total de Síntomas Positivos.

Med Segur Trab (Internet) 2011; 57 (225) 361-370

Palabras claves: Estrés Laboral, Estrés de Rol, Conflicto de Rol, Ambigüedad de Rol.

Abstract

The role stress is specifically the one that originates from the performance of roles in the company, and includes both role ambiguity and role conflict.

Objetive: To evaluate the ambiguity and the role conflict of a group of professionals who carry out different jobs, and its influence on their physical and psychological well-being.

Material and method: To evaluate the role stressors (Role Ambiguity and Role Conflict) the Questionnaire of Rizzo, House and Lirtzman has been used and to establish the consequences the Questionnaire of Symptoms SLC-90-R. 
Results: $20 \%$ of the sample percieved ambiguity and conflict of role in their job and had high punctuation in all the subscales: Somatization, Obsessive- Compulsive, Interpersonal Sensibility, Depression, Anxiety, Hostility and Phobic Anxiety. In addition, the Global Rate of Gravity and the Total Positive Symptoms were significantly higher in the group that had high punctuation in ambiguity of role and conflict of role.

On the other hand, we did not find significant differences in the level of studies in the subscales ,nor the General Rate of Gravity nor in the Total Positive Symptoms.

Med Segur Trab (Internet) 2011; 57 (225) 361-370

Key words: Work Stress, Role Stress, Role Conflict, Role Ambiguity. 


\section{INTRODUCCIÓN}

La investigación sobre conflicto y ambigüedad de rol comienza a principios de los años 50, aunque es en la década de los 60 cuando adquiere verdadero protagonismo, debido fundamentalmente al desarrollo de las teorías sobre el estrés de rol (Kanh y otros, 1964). En las dos décadas siguientes los trabajos realizados en este campo se centran en el estudio de las consecuencias negativas que el conflicto y la ambigüedad de rol tienen para el sujeto y la empresa (insatisfacción laboral, absentismo laboral, rotación, etc...). En concreto, se puso de manifiesto la relación entre el conflicto y la ambigüedad de rol y la insatisfacción laboral, la ansiedad, el bajo rendimiento, el absentismo y la propensión al abandono del puesto (Meliá y otros, 1987). En la actualidad, algunas condiciones del trabajo como el trabajo en grupo, la mayor o menor autonomía, las altas tasas de rotación, etc., hacen que este tema tenga gran interés.

El estrés de rol es específicamente el que se origina por el desempeño de roles en la organización, y comprende tanto la ambigüedad de rol como el conflicto de rol y la sobrecarga de rol (acumulación de deberes y demandas por el desempeño de uno o varios roles) tanto cuantitativa como cualitativa (Miles y Perreault, 1976).

El trabajador con ambigüedad de rol vive en la incertidumbre, no sabe qué se espera de él (Ironson, 1992) (Sandman, 1992), es decir, no tiene configurado con claridad cuál es su rol en la empresa. La ambigüedad de rol se refiere a la situación que vive la persona cuando no tiene suficientes puntos de anclaje para desempeñar su labor o bien éstos no son adecuados; es decir, a la falta de definición por información incompleta, poco concisa, muy cambiante sobre objetivos del trabajo, responsabilidades, comunicación, relaciones de autoridad y procedimientos (Peiró y otros, 1985).

El conflicto de rol se produce cuando hay demandas, exigencias en el trabajo que son entre sí incongruentes o incompatibles para realizar el trabajo (Ironson, 1992) (Sandman, 1992), por expectativas divergentes dentro de la propia organización, por incompatibilidad temporal, por conflicto con el propio sistema de valores y creencias y por conflicto entre los distintos roles individuales (Peiró, 2005). Otros han definido el conflicto de rol como la presencia simultánea de expectativas contradictorias asociadas a un rol (Miles, 1977) (Van Sell y otros, 1981).

Un rol puede ser definido como el conjunto de expectativas y demandas sobre las conductas que se esperan de la persona que ocupa una determinada posición (persona focal). Esas expectativas y demandas son emitidas por los miembros del conjunto de rol, que incluye a todas las personas y/o grupos que son afectados de alguna manera por la conducta de la persona focal y tienen o pretenden tener, de uno u otro modo, la capacidad de influir sobre la conducta de aquella emitiendo expectativas y demandas para ello. Es decir, un rol es un haz de expectativas propias y ajenas acerca del patrón de conductas que se adecua al puesto ocupado (Infiestas Gil, 1991). Así pues, el rol se elabora y se desempeña en el marco de la interacción social que protagonizan la personal focal y los emisores de rol que componen el conjunto de rol (Peiró y González-Romá, 1991).

Cuando los miembros del conjunto de rol envían expectativas con información insuficiente, se produce ambigüedad de rol, y cuando los miembros del conjunto de rol envían a la persona focal demandas y expectativas incompatibles entre sí, se da conflicto de rol.

Se distinguen varios tipos de conflicto de rol: intra-emisor (un mismo emisor presenta demandas incompatibles), interemisores (las demandas de un emisor son incompatibles con las de otro), inter-roles (demandas incompatibles de emisores de distintos roles que desempeña una misma persona) y persona-rol (demandas de los emisores incompatibles con los propios valores personales) (Kahn y otros, 1964). También se ha diferenciado entre conflicto de rol interno (cuando se le propone al trabajador algo que está en contra de sus valores o que considera que no corresponde a su rol) y conflicto de rol externo que se produce cuando dos superiores imponen órdenes o indicaciones opuestas, si se cumple la de uno no se cumple la de otro) (Frías Azcárete, 2005). 
Pero lo que posibilita y favorece que en las organizaciones se produzca la ambigüedad de rol y el conflicto de rol es el constante proceso de evolución y ajuste de roles dentro de ellas y lo que se ha venido en llamar roles limítrofes, tanto internos como externos a la organización (Miles, 1980) (González-Romá y otros, 1989).

Además, aunque desde diversas disciplinas (psicología, psiquiatría, sociología, epidemiología...), va creciendo la investigación sobre las condiciones en el ambiente laboral, es necesario ir precisando y clarificando la terminología porque todavía no se cuentan con definiciones globalmente aceptadas, sobre los conceptos que hacen referencia a los riesgos psicosociales en el trabajo.

\section{OBJETIVO}

El objetivo de este estudio es evaluar la ambigüedad y el conflicto de rol de un grupo de profesionales que ocupan diferentes puestos de trabajo, y su influencia sobre sus niveles de bienestar físico y psicológico. Y asimismo a contribuir a clarificar la fragilidad diagnóstica que existe sobre el estrés laboral y a imprecisas definiciones conceptuales sobre la constelación de todo aquello que tiene cierto grado de relación con lo relativo a lo psicosocial del trabajo.

\section{MATERIAL Y MÉTODO}

\section{Muestra}

Para la realización del estudio se contó con una muestra aleatoria de 60 sujetos, trabajadores del Ayuntamiento de Madrid, Titulados de Grado Medio y Titulados de Grado Superior y de diferentes Servicios y Áreas de trabajo y con un rango de edad entre 28 y 59 años. Se presentan en la tabla I las características sociodemográficas de la muestra.

Tabla I. Características sociodemográficas de la muestra; $n=60$

\begin{tabular}{lcc}
\hline \multicolumn{2}{c}{ Sexo } & Porcentaje \\
\hline \multicolumn{3}{c}{ Edad } \\
Mujer & $60 \%$ \\
\hline $21-30$ & $40 \%$ \\
\hline $31-40$ & \\
$41-50$ & $15 \%$ \\
$51-60$ & Estado Civil & $30 \%$ \\
& & $35 \%$ \\
\hline \multicolumn{2}{c}{ Soltero } & $20 \%$ \\
\hline Casado & $32 \%$ \\
Separado / Divorciado & $42 \%$ \\
Viudo & $24 \%$ \\
\hline & Nivel educativo \\
\hline Universitario grado medio & $2 \%$ \\
\hline Universitario grado superior & $62 \%$ \\
\hline
\end{tabular}




\section{Instrumentos}

Para la recogida de datos se elaboró un cuestionario sociodemográfico (sexo, edad, estado civil y nivel educativo). Para evaluar los estresores de rol (Ambigüedad de Rol y Conflicto de Rol) se ha utilizado la adaptación española del Cuestionario de Rizzo, House y Lirtzman (1970), compuesto por dos escalas que consisten en bloques de frases. Un bloque está formado por seis frases que hacen referencia a la claridad de rol y el otro, de ocho frases, se refiere al conflicto de rol. Y para establecer las consecuencias del estrés de rol el Cuestionario de Síntomas SLC-90-R (Derogatis, 1983). El SCL-90-R es un inventario multidimensional de autoevaluación de síntomas, diseñado para medir el malestar psicológico general a partir de síntomas relacionados con varias dimensiones psicopatológicas y consta de 90 ítems. Las subescalas incluyen las dimensiones sintomáticas de Somatización, Obsesión-Compulsión, Sensibilidad Interpersonal, Depresión, Ansiedad, Hostilidad, Ansiedad Fóbica, Ideación Paranoide y Psicoticismo.

\section{Procedimiento}

Tras seleccionar la muestra de trabajadores del Ayuntamiento. A todos ellos se les administraron los dos cuestionarios y se les garantizó en todo momento la confidencialidad y el anonimato.

Se establecieron dos grupos: Uno, con aquellos que puntuaron alto en ambigüedad y conflicto de rol (grupo con A y C) y otro con los que puntuaron bajo en ambigüedad y conflicto de rol (grupo sin A y C). Y después, se han comparado los resultados de ambos grupos con las puntuaciones obtenidas en las subescalas del Cuestionario de Síntomas SLC-90-R, así como en el Índice Global de Gravedad (sumatorio de todos los ítems) (puntuación total) y en el Total de Síntomas Positivos (frecuencia o número de síntomas con puntuación mayor de cero).

Para las diferencias de medias entre ambos grupos se ha utilizado la prueba t de Student, que se ha realizado a través del paquete estadístico SPSS 12.0.

Tabla II. Características sociodemográficas del grupo con A y C; $n=12$

\begin{tabular}{lcc}
\hline \multicolumn{2}{c}{ Sexo } & Porcentaje \\
\hline Hombre & & $61 \%$ \\
Mujer & Edad & $39 \%$ \\
\hline \multicolumn{2}{c}{} & $20 \%$ \\
\hline $31-40$ & Estado Civil & $35 \%$ \\
$41-50$ & $30 \%$ \\
$51-60$ & $15 \%$ \\
\hline Soltero & \\
Casado & $30 \%$ \\
Separado / Divorciado & $45 \%$ \\
Viudo & $25 \%$ \\
\hline & Nivel educativo & $0 \%$ \\
\hline Universitario grado medio & $65 \%$ \\
Universitario grado superior & $35 \%$ \\
\hline
\end{tabular}




\section{RESULTADOS}

De la muestra de 60 sujetos a los que se les administró el cuestionario de Rizzo, House y Lirtzman (1970), 12 puntuaron alto en Ambigüedad y Conflicto de Rol, y todos los que puntuaron alto en Ambigüedad de Rol, lo hicieron asimismo en Conflicto de Rol y viceversa, ya que existe una correlación significativamente positiva, con un valor mínimo de significación estadística de 0,01 (tabla III), lo que indica que la ambigüedad y el conflicto de rol se solapan o van unidas, o quizá los instrumentos de evaluación de que se disponen para evaluar la Ambigüedad y el Conflicto de Rol no son muy precisos y posibilita que sean superpuestos o aglutinados. Y 48 sujetos de la muestra puntuaron bajo en dichos conceptos. Éstos formaron el grupo de sin Ambigüedad y Conflicto de Rol (tablas IV y V). Lo que viene a indicar que el 20\% de la muestra percibía ambigüedad y conflicto de rol en su trabajo.

Tabla III. Correlación pearson

\begin{tabular}{lc}
\hline & GRUPO CON AMBIGÜEDAD \\
\hline GRUPO CON CONFLICTO DE ROL & 0,99 \\
\hline
\end{tabular}

$\mathrm{p}<0,05$.

Tabla IV. Características sociodemográficas del grupo $\sin$ A y C; $\mathbf{n}=48$

\begin{tabular}{lcc}
\hline & Sexo & Porcentaje \\
\hline & & \\
\hline Hombre & Edad & $41 \%$ \\
Mujer & & \\
\hline & & $10 \%$ \\
\hline $21-30$ & $25 \%$ \\
$31-40$ & $35 \%$ \\
$41-50$ & $30 \%$ \\
$51-60$ & Estado Civil & $34 \%$ \\
\hline Soltero & $40 \%$ \\
Casado & $24 \%$ \\
Separado / Divorciado & $2 \%$ \\
\hline Viudo & & \\
\hline & Nnivel educativo & $60 \%$ \\
\hline Universitario grado superior & \\
\hline
\end{tabular}


Tabla V. Medias y desviaciones típicas

\begin{tabular}{l|r|r|r|r|r}
\hline \multirow{2}{*}{} & \multicolumn{2}{|c|}{$\begin{array}{c}\text { GRUPO CON } \\
\text { A y C n=12 }\end{array}$} & \multicolumn{2}{c|}{$\begin{array}{c}\text { GRUPO SIN } \\
\text { A y C } \mathbf{n = 4 8}\end{array}$} & \\
\cline { 2 - 6 } & \multicolumn{1}{|c|}{ Media } & \multicolumn{1}{c|}{ DT } & Media & \multicolumn{1}{c}{ DT } & \multicolumn{1}{c}{ T } \\
\hline SOMATIZACIÓN & 25,3 & 6,8 & 10,28 & 7,23 & 1,78 \\
OBSESIÓN - COMPULSIÓN & 25,5 & 6,9 & 16,05 & 10,07 & 2,4 \\
SENSIBILIDAD INTERPERSONAL & 34,8 & 3,8 & 15,4 & 9,9 & 0,43 \\
DEPRESIÓN & 32,0 & 7,6 & 9,7 & 7,0 & 1,2 \\
ANSIEDAD & 33,3 & 7,6 & 3,55 & 1,99 & 1,38 \\
HOSTILIDAD & 35,9 & 9,9 & 17,7 & 4,6 & 0,71 \\
ANSIEDAD FÓBICA & 30,04 & 7,9 & 16,4 & 9,0 & 2,12 \\
IDEACIÓN PARANOIDE & 2,2 & 2,2 & 1,2 & 2,2 & 2,7 \\
PSICOTICISMO & 3,01 & 4,11 & 1,3 & 2,2 & 1,5 \\
ÍNDICE GLOBAL DE GRAVEDAD & 33,96 & 8,71 & 16,75 & 4,4 & $-0,73$ \\
TOTAL DE SÍNTOMAS POSITIVOS & 35,62 & 6,3 & 14,4 & 8,4 & $-5,33$ \\
\hline
\end{tabular}

$\mathrm{p}<0,05$.

Los del grupo que puntuó alto en ambigüedad y conflicto de rol puntuó asimismo alto en todas las subescalas de somatización (refleja el distrés que surge de la percepción de disfunción corporal), de Obsesión-Compulsión (refleja conductas que están íntimamente relacionadas con este síndrome clínico), de Sensibilidad Interpersonal (hace referencia a sentimientos de inadecuación e inferioridad personal, especialmente en comparación con otros individuos. También pone de manifiesto el autodesprecio, y la dificultad y el malestar que surge durante la interacción interpersonal), Depresión, Ansiedad, Hostilidad (existencia de pensamientos, sentimientos y conductas hostiles), Ansiedad Fóbica (recoge tanto síntomas de agorafobia como de algunas fobias específicas frecuentes), Ideación Paranoide y de Psicoticismo (incluye desde comportamientos esquizoides hasta síntomas claramente psicóticos), aunque en estas dos últimas escalas existe menor significación. Además el Índice Global de Gravedad y el Total de Síntomas Positivos fueron significativamente superiores en el grupo que puntuó alto en ambigüedad de rol y conflicto de rol.

\section{DISCUSIÓN Y CONCLUSIONES}

Se ha afirmado que el conflicto de rol presenta relaciones significativas de signo positivo con tensión laboral, ansiedad, depresión relacionada con el trabajo, síntomas neuróticos, quejas somáticas, fatiga, hipertensión, niveles elevados de colesterol, obesidad y enfermedades coronarias; y presenta relaciones significativas de signo negativo con satisfacción laboral, implicación en el trabajo, desempeño, compromiso organizacional, participación y autoestima (Peiró, 2005).

Y la ambigüedad de rol relaciona con tensión, ansiedad, agotamiento emocional, depresión, fatiga, quejas somáticas, propensión a abandonar la organización y sentimientos de resentimiento. Y se relaciona negativamente con satisfacción laboral, sentimientos de competencia, implicación en el trabajo, autoestima, autoconfianza, calidad en la toma de decisiones y participación (Mclean, 1974) (Peiró, 2005).

Y tanto el conflicto de rol como la ambigüedad de rol se han relacionado con la insatisfacción laboral (Meliá y otros, 1987).

Por otro lado, se ha apuntado como factores causantes de burnout al conflicto de rol y a la ambigüedad de rol (Zellars y otros, 1999), aunque este compromiso tiene cierta debilidad, ya que para que se dé el síndrome de burnout tiene darse además 1 requisito 
específico de profesional que mantiene un contacto constante y directo con personas que son beneficiarios del propio trabajo

A pesar de que el concepto de estrés de rol es utilizado con cierta inespecificidad (Osca y otros, 2003) sería conveniente establecer el diagnóstico de estrés de rol únicamente para aquella sintomatología derivada del conflicto de rol y de la ambigüedad de rol, para ir clarificando los conceptos y definiciones conceptuales imprecisas, ya que como se deduce de los resultados de este trabajo la ambigüedad de rol y el conflicto de rol se solapan o van unidas. La sobrecarga de rol tanto cuantitativa como cualitativa también debería incluirse bajo estrés de rol. y no debería subsumirse como un tipo de conflicto de rol como realizan Khan y otros, 1964, sino como entidad diferente del mismo orden que la ambigüedad de rol y del conflicto de rol como también señala la Comisión Nacional del Sida en su Documento Técnico de Apoyo sobre estrés laboral (2001).

Y dejar exclusivamente el concepto y diagnóstico de estrés laboral, que con frecuencia es utilizado como un cajón de sastre, para hacer referencia a lo relativo los estresores del ambiente físico (iluminación, ruido temperatura...), al ritmo de trabajo, nivel de autonomía, monotonía, carga física, carga mental, horario de jornada laboral, turnos y sobrecarga laboral.

En el análisis de las diferencias entre el grupo con A y C y el grupo sin A y C. El grupo con A y C tiene puntuaciones significativamente más altas en todas las subescalas: Somatización, Obsesión-Compulsión, Sensibilidad Interpersonal, Depresión, Ansiedad, Hostilidad, Ansiedad Fóbica, Ideación Paranoide y Psicoticismo

Además con respecto a las diferencias de sexo en el grupo con A y C (tabla VI), los hombre puntúan más alto en Hostilidad y Ansiedad Fóbica, y las mujeres en Somatización, Obsesión-Compulsión, Sensibilidad Interpersonal, Depresión y Ansiedad. Asimismo los hombres puntuaron más alto tanto en el Índice General de Gravedad pero no hubo diferencias significativas en el Total de Síntomas Positivos. En ningún caso se encuentra significación estadística con respecto a Ideación Paranoide y Psicoticismo.

Tabla VI. Medias y desviaciones típicas del grupo con A y C (diferencias entre sexo)

\begin{tabular}{l|r|r|r|r|r}
\hline \multirow{2}{*}{} & \multicolumn{2}{|c|}{ HOMBRES } & \multicolumn{2}{c|}{ MUJERES } & \\
\cline { 2 - 6 } & Media & DT & Media & \multicolumn{1}{c}{ DT } & T \\
\hline SOMATIZACIÓN & 26,43 & 5,8 & 9,8 & 7,3 & 2,56 \\
OBSESIÓN - COMPULSIÓN & 27,5 & 4,9 & 6,5 & 10,7 & 2,49 \\
SENSIBILIDAD INTERPERSONAL & 36,8 & 5,8 & 10,4 & 9,4 & 1,37 \\
DEPRESIÓN & 33,2 & 2,6 & 8,7 & 7,3 & 1,65 \\
ANSIEDAD & 32,3 & 6,6 & 3,5 & 2,9 & 3,22 \\
HOSTILIDAD & 35,9 & 8,9 & 7,7 & 5,6 & 1,27 \\
ANSIEDAD FÓBICA & 34,4 & 5,9 & 6,4 & 9,2 & 1,83 \\
IDEACIÓN PARANOIDE & 34,2 & 4,2 & 3,2 & 2,7 & 0,23 \\
PSICOTICISMO & 0,5 & 5,2 & 1,3 & 2,9 & 3,22 \\
ÍNDICE GLOBAL DE GRAVEDAD & 21,8 & 9,1 & 0,75 & 4,7 & $-1,26$ \\
TOTAL DE SÍNTOMAS POSITIVOS & 36,6 & 5,4 & 4,4 & 7,4 & $-1,51$ \\
\hline
\end{tabular}

$\mathrm{p}<0,05$.

Por otro lado, no se encuentran diferencias significativas entre los niveles de estudios ni en las subescalas ni en Índice General de Gravedad ni en el Total de Síntomas Positivos (tabla VII). 
Tabla VII. Medias y desviaciones típicas del grupo con A y C (diferencias entre nivel educativo)

\begin{tabular}{l|c|c|c|c|c}
\hline \multirow{2}{*}{} & \multicolumn{2}{|c|}{$\begin{array}{c}\text { UNIVERITARIO GRADO } \\
\text { MEDIO }\end{array}$} & $\begin{array}{c}\text { UNIVERSITARIO GRADO } \\
\text { SUPERIOR }\end{array}$ & \\
\cline { 2 - 6 } & Media & DT & Media & DT & \multicolumn{1}{c}{ T } \\
\hline SOMATIZACIÓN & 23,3 & 6,8 & 11,2 & 6,3 & 0,67 \\
OBSESIÓN - COMPULSIÓN & 24,5 & 6,9 & 14,5 & 8,7 & 0,21 \\
SENSIBILIDAD INTERPERSONAL & 34,8 & 4,8 & 14,4 & 9,9 & 0,22 \\
DEPRESIÓN & 32,0 & 7,6 & 9,7 & 7,2 & 0,65 \\
ANSIEDAD & 33,3 & 7,6 & 3,5 & 1,9 & 2,22 \\
HOSTILIDAD & 36,9 & 9,9 & 16,7 & 4,6 & 1,79 \\
ANSIEDAD FÓBICA & 30,4 & 7,9 & 16,4 & 9,4 & 1,30 \\
IDEACIÓN PARANOIDE & 1,2 & 3,2 & 1,2 & 2,1 & 0,33 \\
PSICOTICISMO & 2,1 & 4,1 & 1,3 & 1,2 & 0,49 \\
ÍNDICE GLOBAL DE GRAVEDAD & 33,6 & 5,7 & 15,5 & 4,4 & $-1,79$ \\
TOTAL DE SÍNTOMAS POSITIVOS & 35,6 & 4,3 & 13,4 & 7,4 & $-1,30$ \\
\hline
\end{tabular}

$\mathrm{p}<0,05$.

De manera que los datos obtenidos y analizados permiten pensar que las consecuencias del estrés de rol en el trabajo tiene influencia sobre sus niveles de bienestar físico y psicológico.

\section{REFERENCIAS BIBLIOGRÁFICAS}

Comisión Nacional del Sida. Estrés Laboral y Burnout en los Servicios de Salud. Documento Técnico de Apoyo. Área de Prevención. Madrid. 2001

Derogatis, L.R. Cuestionario de Síntomas SLC-90-R (versión española de González de Rivera y de las Cuevas, 1988) TEA. Madrid. 1983.

Frías Azcárate, R. www. ucm.es/7nfo/eurotheo/diccionario/S/stress.htm.2005.

Gonzalez-Roma, V.; Sancerni, M.D.; Meliá, J.L.; Tomás, J.M.; Oliver, A. Una escala para medir la limitrocidad organizacional. Trabajo presentado en la II Conferencia Española de Biometría. Segovia.1989.

Infiestas GIL, A. Sociología de la empresa. Amar Ediciones. Salamanca. 1991.

Ironson, G.H. Jobstress and Health. En C.J. Cranny, P.C. Smith, E.F. Stone. Job Satisfaction. Lexington Books. New York. 1992

Kahn, R.L.; Wolfe, D.M.; Quinn, R.P.; Snoek, J.D. Rosenthal, R.A. Organizational Stress: Studies in Role Conflict and Ambiguity. New York. John Wiley and Sons.1964.

McLean, A. Work Stress. Addison Wesley Publising Company. USA. 1974

Meliá, J.L.; Zornoza, A.; Sanz, M.J.; Morte, M.P.; Gonzalez, V. La incidencia de los factores de conflicto de rol y ambigüedad de rol sobre los factores de la satisfacción laboral. Actas del Segundo Congreso nacional de Evaluación Psicológica. Madrid. 1987.

Miles, R. H. Role-set configuration as a predictor of role conflict and ambiguity in complex organizations. Sociometry. 40, 1, 21-34. 1977.

Miles, R. H. Boundary Roles. En C.L. Cooper y R. Payne. Current Concerns in Occupational Stress. Chichester, John Wiley and Sons. 61-98. 1980.

Miles, R. H.; Perreault, W. D. Organizational role conflict: its antecedents and consequences. Organizational Behavior and Human Performance. 17. 19-44. 1976.

Osca, A.; González-Camino, G.; Bardera, P.; Peiró, J.M. Estrés de rol y su influencia sobre el bienestar psíquico y físico en soldados profesionales. Psicothema. 15, 1, 54-57. 2003

Peiró, J.M.; Meliá, J.L.; Gonzalez, V. La incidencia del conflicto y ambigüedad de rol sobre la propensión al abandono y la satisfacción laboral. Actas del II Congreso Nacional de Psicología del Trabajo. Barcelona. 305-333. 1985 
Peiró, J.M. Desencadenantes del Estrés Laboral. Ediciones Pirámide. Madrid. 2005.

Peiró, J. M.; González-Romá, V. Role-set variables as antecedent of role stress: a causal model. Revue International de Psychologie Sociale. 4, 29-44. 1991.

Rizzo, J.; House, R.E.; Lirtzman, J. Role conflict and ambiguity in complex organizations. Administrative Science Quarterly. 15, 150-163. 1970.

Sandman. B.A. The measurement of job stress. En C.J. Cranny, P.C. Smith, E.F. Stone. Job Satisfaction. Lexington Books. New York. 1992.

Zellars, K.L.; Perrewe, P.L.; Hochwarter, W.A. Mitigating burnout among high-NA employees in health care: what can organizations do?. Journal of Applied Social Psychology. 29, 11, 2250-2271. 1999.

Van Sell, M.; Brief, A.P.; Schuler, R.S. Role conflict and role ambiguity: Integration of the literature and directions for future research. Human Relations. 1, 43-71. 1981.

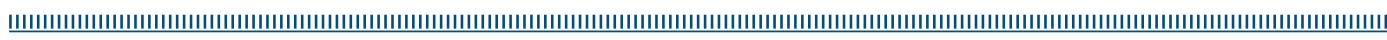

